\title{
URETERORRENOSCOPIA (URS) FLEXIBLE: TÉCNICA Y RESULTADOS.
}

\author{
José Ramón Cansino Alcaide, Javier Reinoso Elbers, David López Sánchez, Sonia Pérez \\ González, Alfredo Aguilera Bazán, Alberto Rando Tous, Luís Hidalgo Togores y Javier De La \\ Peña Barthel.
}

Servicio de Urología. Hospital Universitario La Paz. Madrid. España.

\begin{abstract}
Resumen.- OBJETIVO: Los objetivos fundamentales de éste trabajo son dos. Por una parte exponer la técnica empleada en nuestro Servicio ya que difiere en algunos aspectos importantes de las publicadas en otros centros, explicando en algunos casos pequeños detalles que pueden ayudar al mejor desarrollo de la técnica. Por otra parte, exponemos los resultados de una serie de 100 casos.

MÉTODOS: Presentamos nuestra serie reciente de URS Flex para el tratamiento de la litiasis renal. Para ello hemos revisado de forma retrospectiva éste tratamiento desde Enero de 2007 hasta Marzo de 2010. El tamaño medio de la litiasis tratada es de $1.5 \mathrm{~cm}(0.5-6 \mathrm{cms})$ y en todos los casos utilizamos vainas protectoras del
\end{abstract}

\section{CORRESPONDENCIA}

José Ramón Cansino Alcaide

Servicio de Urología Hospital Universitario La Paz

Pso. de la Castellana, 261

28046 Madrid (España)

urocansino@yahoo.es

Aceptado para publicar: 15 de octubre 2010 ureteroscopio. La litotricia empleada en todos los casos fue láser de holmio con fibras de 200 y 365 micras

RESULTADOS: El porcentaje de pacientes que quedaron libres de litiasis (stone free rate-SFR) tras la cirugía en el postoperatorio inmediato fue de 77/100 pacientes (77\%) contabilizando como resto litiásico fragmentos visualizados mediante la fluoroscopia del quirófano y la visión directa del URS Flex. A los 3 meses de la cirugía 89/96 pacientes (92.7\%) estaban libres de litiasis comprobado mediante urografía intravenosa.

Respecto a las complicaciones destacar 5 pacientes con lesión ureteral durante la colocación de la vaina protectora y 9 pacientes que acudieron a urgencias en el postoperatorio por molestias secundarias al catéter doble J.

CONCLUSIÓN: Como conclusión podemos defender el tratamiento de las litiasis renales mediante URS Flex hasta un tamaño máximo de $2 \mathrm{~cm}$ siguiendo nuestro algoritmo terapéutico.

Palabras clave: Ureteroscopia flexible. Litiasis. Endourología.

Summary.- OBJECTIVES: This paper has two main objectives: First, to expose the URS technique used in our Department because there are important differences with respect to others centres published, explaining same aspect that can benefit to the better development of the technique. Second, we present the results of a series of 100 cases. Flexible ureterorenoscopy (Flex URS) has been little used to date, mainly because of the technical difficulties created by the deficient quality of the instruments used, such as ureteroscopes offering scant visibility, poor illumination, a small working channel, deficient quality of the forceps and baskets, etc. 
METHODS: We present our recent series of flexible URS for the treatment of renal lithiasis.

We performes a retrospective analysis of this treatment corresponding to the period between January 2007 and March 2010. In this period we have treated 100 patients.

The medium size of the stone treated is $1.5 \mathrm{~cm}(0.5-6 \mathrm{~cm})$ and we used ureteroscopic protector sheath in all cases. The lithotripter system used in all cases was Ho:YAG Laser with 200 and 365 micras fibers

RESULTS: The stone-free rate (SFR) in the immediate postoperative period was 77\% (77/100 patients). Residual stones, defined as stone fragments visualized in the operating room via fluoroscopy and directly with the flexible ureteroscope.

Three months after surgery, the SFR was 92.7\% 189/96 patients) confirmed by intravenous urography. Regarding complications, we had 5 patients with ureteral lesions during protector sheath pass and 9 patients that presented at the emergency room with pain secondary to the double I catheter.

CONCLUSIONS: Flexible URS for of renal lithiasis can be defended in stones measuring up to $2 \mathrm{~cm}$ in diameter, based on our treatment algorithm.

Keywords: Flexible ureterorenoscopy. Lithiasis. Endourology.

\section{INTRODUCCIÓN}

La ureterorrenoscopia flexible (URS Flex.) ha sido una técnica poco empleada hasta la fecha, debido fundamentalmente a la dificultad técnica por la mala calidad de los instrumentos con los que teníamos que trabajar, como ureteroscopios con mala visibilidad, poca luz, pequeño canal de trabajo, poca y mala calidad de pinzas y cestillos lo que limitaba mucho la vida media de éstos aparatos y por tanto suponía un elevado coste para el servicio, máxime, si era utilizado por varios urólogos.

Los inicios de la URS Flex los encontramos en 1964 con una publicación de Marshall (1) donde describe por primera vez una exploración endoscópica del tracto urinario superior, pero es Takayasu en 1971 el que lo realiza con un instrumento flexible y lo documenta por primera vez (2). Posteriormente han de pasar casi 20 años hasta que comencemos a ver con más frecuencia artículos sobre ésta técnica, gracias al desarrollo de nuevos ureteroscopios, como los de Bagley $(3,4)$ en 1987, quien incluso nos propuso unas guidelines sobre la URS Flex.
Es en los años 90 y 2000 cuando verdaderamente se ha convertido en un abordaje importante con aplicación en muchos centros como lo demuestran las numerosas publicaciones al respecto en éstos años incluso para el tratamiento de litiasis coraliformes (5).

En la última década y sobretodo en los últimos 5 años con la aparición de nuevos URS flex más resistentes y con mejor calidad de imagen, el desarrollo del láser y sus fibras de finos calibres y una gran variedad de cestillos que permiten una flexión y deflexión máxima del URS, ésta técnica ha sufrido un verdadero renacer, ampliando su uso, indicaciones y posibilidades.

\section{MATERIAL Y MÉTODOS}

Presentamos nuestra serie reciente de URS Flex para el tratamiento de la litiasis renal.

Para ello hemos revisado de forma retrospectiva éste tratamiento desde Enero de 2007 hasta Marzo de 2010.

Todas las cirugías fueron realizadas por dos urólogos del Servicio (R.C. y L.H.) ambos con amplia experiencia previa en endourología.

Desde Enero 2007- Febrero 2010 hemos tratado a 100 pacientes con una media de edad de 51,7 años (24-84).

A todos los pacientes se les realizó urografía intravenosa de forma preoperatoria para determinar tamaño y localización de la litiasis, así como la valoración del abordaje mediante URS Flex.

En todos los casos se utilizaron vainas protectoras del URS Flex (Cook Urological Flexor ${ }^{\circledR} 12$ $14 \mathrm{Fr}$; Boston Scientific Microvasive Urology NavigatorTM 11-13Fr; ACMI UroPass ${ }^{\circledR}$ 12-14Fr).

Para la mayoría de los procedimientos empleamos un URS Flex Wolf diámetro exterior 9 charrier y ocasionalmente (menos de 10 cirugías) Gyrus ACMI's digital ureterosrenoscope DUR-D.

El $57 \%$ de los pacientes eran hombres y el $43 \%$ eran mujeres siendo la litiasis a tratar en 48 pacientes del lado izquierdo y 52 pacientes del lado derecho.

El tamaño de la litiasis osciló entre $0.5-6 \mathrm{cms}$ con una media de $1,5 \mathrm{cms}$. 
Se trataron 128 litiasis en 100 pacientes con la siguiente distribución anatómica: $95(74,21 \%)$ en cáliz inferior, $23(17,96 \%)$ en cáliz medio, 8 (6,25\%) en cáliz superior y dos pacientes $(1,56 \%)$ con litiasis coraliformes incompletos (Tabla I).

Una de las pacientes tratadas presentaba una litiasis de $4 \mathrm{cms}$ desde cáliz inferior a pelvis renal y otra de $2 \mathrm{cms}$ en cáliz superior (Masa litiásica total tratada y contabilizada en base de datos como de $6 \mathrm{cms}$ ) que fue tratada con éxito en un solo acto quirúrgico.

La composición mayoritaria de las litiasis por pacientes es la siguiente:

- Oxalato cálcico monohidrato 22

- Oxalato cálcico dihidrato 29

- Fosfato amónico magnésico 18

- Fosfato cálcico 12

- Ácido úrico 18

- Cistina 1

\section{TÉCNICA}

La posición del paciente no debe ser una litotomía muy forzada para facilitar el paso de la vaina protectora y del URS Flex de una forma sencilla y lo más en línea recta posible.

Comenzamos habitualmente con el paso de un alambre guía a través del orificio ureteral hasta llegar a pelvis renal o cáliz superior y visualizamos la litiasis con control fluoroscópico.

TABLA I.

\begin{tabular}{|l|c|}
\hline & № litiasis \\
\hline Cáliz inferior & $95(74,21 \%)$ \\
\hline Cáliz medio & $23(17,96 \%)$ \\
\hline Cáliz superior & $8(6,25 \%)$ \\
\hline Coraliforme & $2(1,56 \%)$ \\
\hline
\end{tabular}

Posteriormente y a través del alambre guía, pasamos un catéter balón de dilatación (usamos Boston o Cook en la mayoría de los casos y según disponibilidad en el Servicio) para dilatar hasta 15 Fr desde el orificio ureteral hasta uréter ilíaco en los casos que sea necesario. Esto es debido a que la vaina protectora suele tener un calibre externo de 1314-15 Fr aunque los nuevos URS Flexibles más finos, permiten un calibre menor.

Introduciremos una sonda vesical 10 ó $12 \mathrm{Fr}$ para asegurar el vaciado vesical durante la cirugía.

Tras dilatar el uréter hasta el cruce ilíaco, realizaremos una ureteropielografía retrógrada que nos ayude a controlar posteriormente el paso de la vaina protectora del URS Flex que utilizamos en todos los casos.

La vaina protectora la pasaremos sobre el alambre guía y quedará alojada en pelvis renal. Posteriormente retiramos el obturador de la vaina protectora con el alambre guía, dejando únicamente la vaina protectora en pelvis renal y la sonda vesical.

El uso de la vaina protectora del URS Flex que usamos en todos los procedimientos, no solo nos ayudará durante la cirugía a mejorar la visibilidad, a mantener bajas presiones intrarrenales o facilitar la extracción de fragmentos sino que además alarga considerablemente la vida media del URS Flex y supone un ahorro económico considerable.

Aunque no usamos nunca un alambre guía de seguridad, éste puede ser de utilidad en algunos casos, por lo que si al cirujano le ofrece mayor seguridad puede ser empleado sin perjudicar el desarrollo de la técnica.

Una vez estemos con el URS Flex dentro de la pelvis renal, intentaremos localizar la litiasis y dirigirla con ayuda de un cestillo a una localización que nos permita trabajar en línea recta como cáliz superior o pelvis renal.

En el interior de un cáliz, tendremos la ventaja adicional de que la litiasis tendrá menor movilidad durante la fragmentación con láser.

Si la litiasis es de un tamaño mayor a 1.5 cms de diámetro, es difícil poderla atrapar con un cestillo debido al diámetro máximo de éstos que suele ser de $1 \mathrm{~cm}$, por lo que nos veremos obligados a iniciar la fragmentación ahí donde esté y luego con fragmentos de menor tamaño podemos elegir llevarlos a otro lugar o seguir trabajando en el mismo sitio si estamos cómodos. 
El trabajar en localizaciones distintas al cáliz inferior no solo es importante para la comodidad durante la litofragmentación trabajando en línea recta, sino que también facilita que los fragmentos litiásicos residuales que puedan quedar, sean expulsados de forma espontánea con mayor facilidad (Figuras 1 y 2).

El calibre de las fibras de láser empleado puede ser de 150-220-360 micras y eventualmente en casos muy seleccionados, la fibra de 550 micras puede ser utilizada en línea recta. Ésta fibra no te permite ningún grado de flexión y forzarlo puede favorecer roturas del URS Flex.

La fibra de 150-220 te permite todos los grados de flexión que tu URS Flex tenga, mejor visibilidad por limitar menos el flujo de lavado con la contrapartida de poder usar menos potencia del Láser y mayor fragilidad y facilidad de rotura de las fibras con su coste adicional.

La fibra de 360 micras es la que usamos en la mayoría de los casos, te limita muy pocos grados y al ser de diámetro mayor, te permite mayor superficie de contacto con la litiasis y por tanto la fragmentación de la litiasis llevará menos tiempo además de resistir mayor potencia de litotricia y ser una fibra más resistente a la rotura por manipulación de la misma. Es importante evitar en la medida de lo posible un medio hemático ya que el canal de lavado de URS Flex y su flujo no permiten una buena visualización.

Si ocurriera esto deberemos aumentar la presión del flujo de entrada sin temor a elevar las presiones intrarrenales gracias a que siempre utilizamos

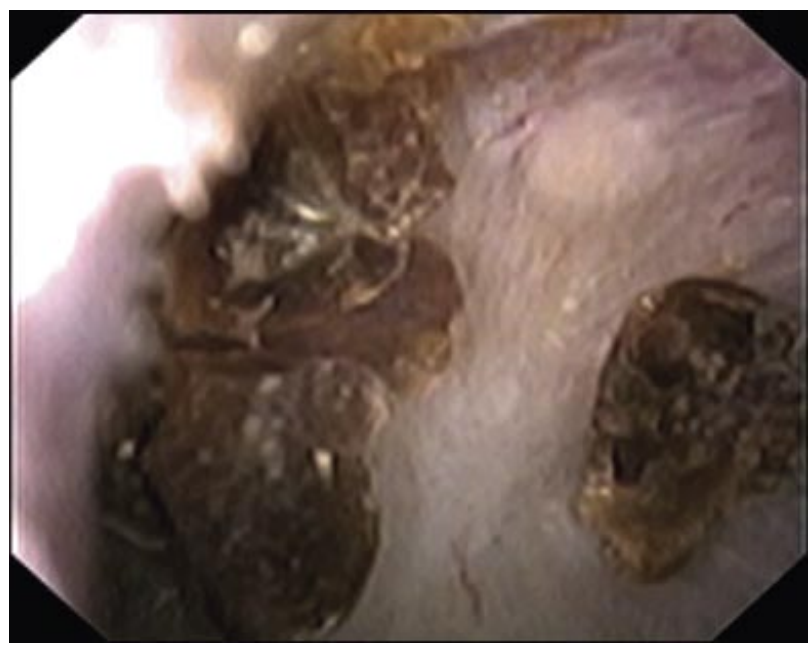

FIGURA 1. Visión de litiasis tras lasertricia. la vaina protectora del URS Flex y mantenemos una presión intrarrenal constante.

Aunque nosotros utilizamos un sistema de irrigación por gravedad, en determinadas ocasiones nos ayudamos de un incremento de presión manual mediante jeringa de $60 \mathrm{ml}$ conectada al URS Flex mediante un sistema de suero salino.

Una vez finalizada la litotricia, para la cual comenzamos con $0.5 \mathrm{~J}$ y $5 \mathrm{~Hz}$ y vamos subiendo la energía del láser en función de la dureza de la litiasis, debemos con ayuda de cestillos de nitinol sin punta, extraer los fragmentos de mayor tamaño a través de la vaina protectora evitando la utilización de pinzas a través del URS Flex que pueden favorecer la rotura del mismo (Figuras 3-5).

Procuramos dejar los menores fragmentos posibles, aunque la filosofía de éste tratamiento es distinto de la Nefrolitotomía Percutánea (NLP).

En el caso de la URS Flex se realiza una litotricia "in situ" por lo que siempre va a quedar polvillo litiásico residual con o sin fragmentos residuales y en mayor cantidad a mayor tamaño de la litiasis tratada. Estos fragmentos residuales de pequeño tamaño o el polvillo litiásico residual, serán expulsados de forma espontánea ayudados del catéter "doble J" que dejamos a la mayoría de los pacientes y medidas posturales con correcta hidratación, similar

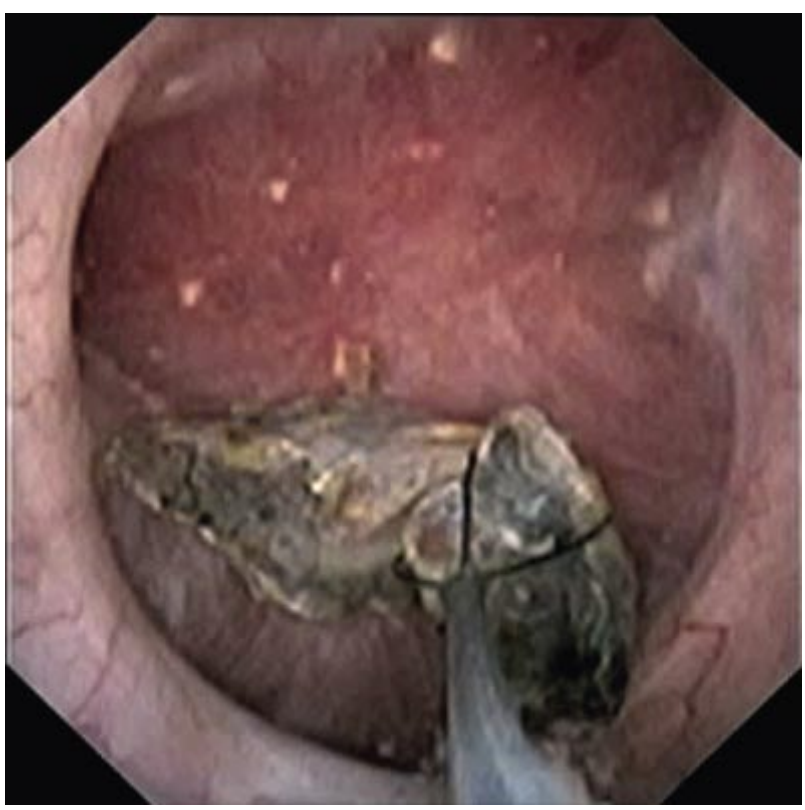

FIGURA 2. Imagen de atrapamiento de la litiasis con dormia. 


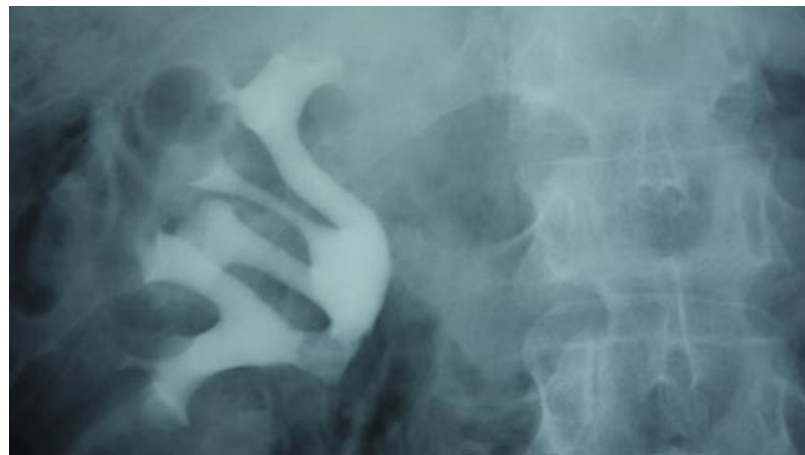

FIGURA 3. Imagen de urografía intravenosa de litiasis tratada con URS Flex.

a lo sucedido tras una sesión de litotricia extracorpórea.

Si la dureza es alta preferimos fragmentar lo justo para extraer el máximo de fragmentos y dejar poco trabajo al aclaramiento litiásico espontáneo. Pero si la litiasis es de baja dureza, podemos dejar fragmentos tras la litotricia "in situ" que se eliminarán de forma espontánea en el postoperatorio con lo que ganamos tiempo durante la cirugía.

En determinados casos el empleo de alfa bloqueantes puede favorecer ésta eliminación y una mejor tolerancia del catéter.

El paciente ha de ser evaluado tras $1-3$ semanas a la cirugía para la retirada del "doble J" o 6 semanas si existió lesión ureteral. Posteriormente a los 3 meses es visto en consulta con una prueba de imagen tipo urografía o Uro-TAC.

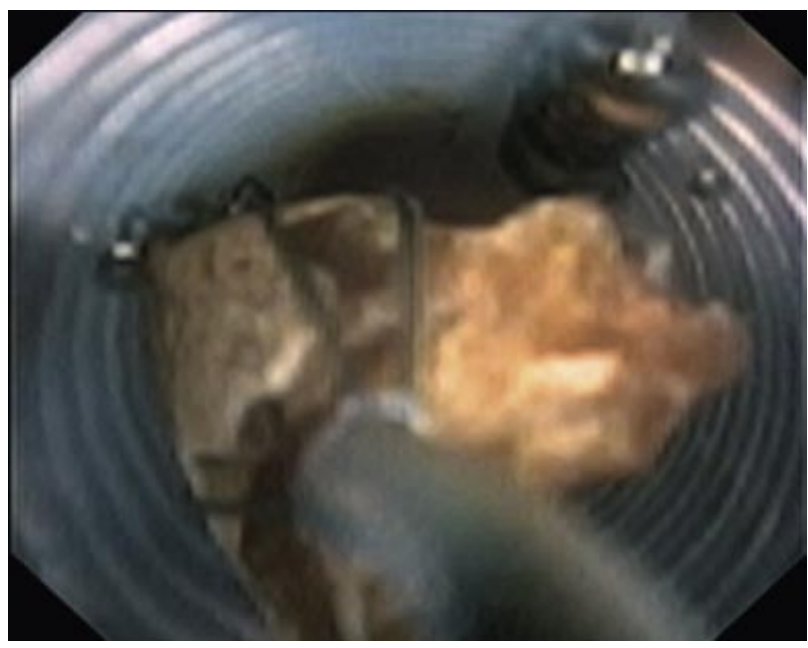

FIGURA 4. Extracción de litiasis a través de la vaina protectora.
Es en éste momento cuando podremos valorar si existe o no litiasis residuales y si precisan algún tipo de tratamiento o no según su clínica.

\section{RESULTADOS}

De todos los pacientes, solo hemos podido ofrecer resultados de porcentaje libre de litiasis en 96, ya que los últimos 4 están pendientes de ser evaluados.

El tiempo quirúrgico fue de 72,3 minutos de media (30-120 $\mathrm{min}$ ) y el sangrado despreciable en todos los pacientes sin necesidad de transfusión.

De los 100 pacientes, en 7 casos se realizó el procedimiento sin suspender la antiagregación plaquetaria por recomendación anestésica.

La litotricia empleada en todos los casos fue Láser de Holmio y se utilizó vaina protectora del ureteroscopio en todos los casos.

La mayoría de los pacientes permaneció tras la cirugía con Pig-tail durante 3 semanas salvo 5 pacientes que lo tuvieron durante 6 semanas por lesión ureteral durante la colocación de la vaina protectora y 2 pacientes que fueron dados de alta sin catéter ya que el calibre ureteral era amplio.

Ningún paciente ha desarrollado estenosis ureteral en éste periodo de tiempo, aunque el $9 \%$

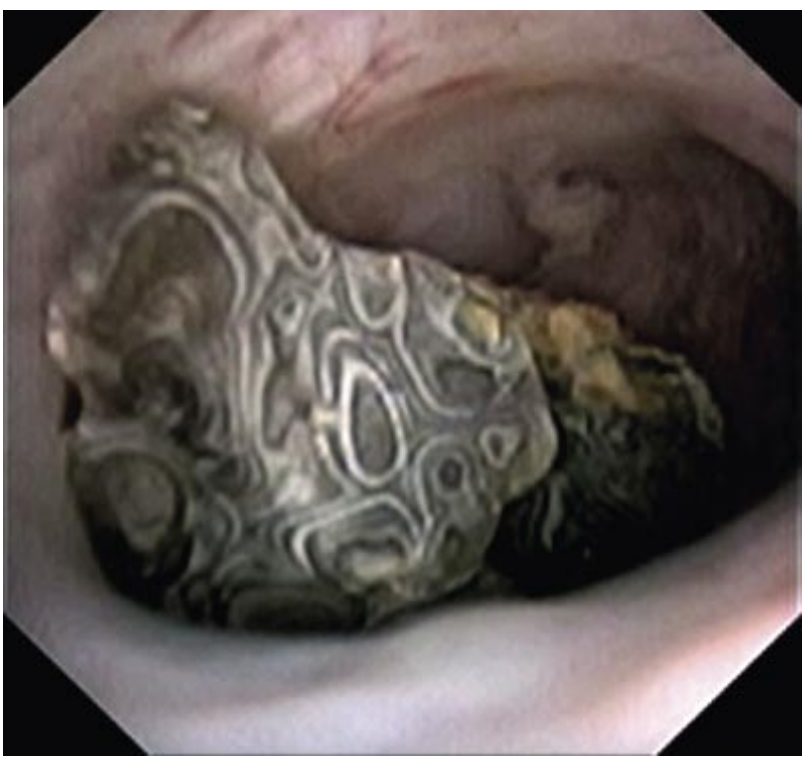

FIGURA 5. Litiasis en cáliz inferior. 
(9 pacientes) acudieron a urgencias en el postoperatorio por molestias debidas al "doble J" y que requirieron tratamiento oral con anticolinérgicos 0 alfa bloqueantes y analgesia convencional sin necesidad de ingreso hospitalario.

El porcentaje de pacientes que quedaron libres de litiasis (stone free rate-SFR) tras la cirugía en el postoperatorio inmediato fue de $77 / 100$ pacientes $(77 \%)$ contabilizando como resto litiásico fragmentos visualizados mediante la fluoroscopia del quirófano y la visión directa del URS Flex.

A los 3 meses de la cirugía 89/96 pacientes $(92.7 \%)$ estaban libres de litiasis comprobado mediante urografía intravenosa.

Los 7 pacientes restantes con litiasis residual están asintomáticos (fragmentos residuales en todos los casos de menos de $0.5 \mathrm{cms}$ ) y ningún paciente hasta la fecha ha requerido un segundo tratamiento.

\section{DISCUSIÓN}

La URS Flex ha ido ganando terreno en los últimos años gracias al desarrollo de las nuevas generaciones de ureteroscopios, la experiencia de los cirujanos y a la eficacia y seguridad de su tratamiento.

Otro avance es el uso sistemático de las vainas protectoras.

Nosotros compartimos la opinión de Preminger (6) a la hora de defender el uso de la vaina protectora del URS Flex que usamos en todos los procedimientos y nos parece un detalle fundamental ya que una de las críticas más encendidas hacia la URS Flex es su vida media y por tanto su elevado coste en reparaciones, problema que se minimiza enormemente con el uso sistemático de éstas vainas.

En caso de necesitar dilatación para introducirlas, preferimos usar los catéteres balón de dilatación ya que aunque pueda suponer un coste adicional, aportan seguridad y disminuyen el riesgo de lesión ureteral en comparación con la dilatación directamente realizada con la vaina protectora del ureteroscopio.

Con el catéter balón realizamos una expansión del calibre y una vez dilatado puede pasar la vaina protectora sin dificultad.

En caso de dilatar directamente con la vaina, ejerceríamos una doble agresión simultánea sobre el uréter como es la dilatación y tracción para ascender la vaina, por lo que creemos que es aplicar una agresión mayor y no siempre se conseguiría ascender la vaina sin lesionar el uréter.

Debemos recordar que los calibres más usados de las vainas protectoras son 12-14 Fr (internoexterno) que es la que usamos en la gran mayoría de los casos, ó 13-15 Fr (interno-externo).

En 2005 Pearle, Lingeman y cols (7) publicaron su serie comparativa con Litotricia extracorpórea (LEOC) de 67 pacientes tratando litiasis de $1 \mathrm{~cm}$ con una tasa libre de litiasis (SFR) del $50 \%$.

Respecto al tamaño de la litiasis a tratar, el límite ha ido ascendiendo a medida que los urólogos han ido ganando experiencia en éste campo y también cómo la tasa de litiasis residual es mayor a medida que tratamos litiasis de mayor tamaño.

Así podemos ver cómo Wong (8) reconocía en 2008 un tamaño límite de 1,5cms en polo inferior para el tratamiento con URS Flex y destacaba el trabajo de los Drs. Grasso y Ficazzola con 90 litiasis tratadas en el polo inferior y $95 \%$ de SFR a los 3 meses de la cirugía con litiasis de menos de $2 \mathrm{cms}$ y $82 \%$ para los mayores de $2 \mathrm{cms}(9)$.

Chung y cols en 2008 reconocían al comparar NLP y URS Flex entre $1-2 \mathrm{cms}$ que tanto el SFR como el número de complicaciones era mayor en NLP pero con diferencias no estadísticamente significativas (10).

En ese mismo año, Breda abría nuevos límites para la URS Flex al publicar su serie de 21 tratamientos de litiasis de hasta $4 \mathrm{cms}$ (11).

EI SFR era de $93,3 \%$ a pesar de necesitar varios procedimientos (hasta 3) y aunque hay otros autores que ofrecen buenos resultados con litiasis de gran tamaño con un SFR de $75-95 \%$ como El-Anay y cols, (12) aparecen también publicaciones contrarias al tratamiento de litiasis de más de $2 \mathrm{~cm}$ con URS Flex como Miller y Lingeman y como Mc Dougall que ofrecen un $95 \%$ de SFR con NLP en un solo procedimiento $(13,14)$.

Breda publicó otra serie en 2009 sobre el tratamiento de múltiples litiasis renales con 51 pacientes y una media de 3,1 litiasis por paciente de $6,6 \mathrm{~mm}$ de media.

Con un tiempo quirúrgico de $65 \mathrm{~min}$ de media el SFR para litiasis de menos de $2 \mathrm{~cm}$ fue del $100 \%$ y en mayores a $2 \mathrm{~cm}$ de $85,1 \%$ (con incluso 2 procedimientos por paciente) (15). 
Mariani presentó su serie de URS Flex con litiasis de más de $4 \mathrm{~cm}$ en pacientes con mucha comorbilidad demostrando que en determinados casos es mejor varias URS que una NLP o una cirugía abierta (16).

En los casos en los que el tamaño de la litiasis es de gran tamaño puede ser de utilidad el uso de la técnica descrita como "popcorn effect "que disminuye el tiempo quirúrgico y es realmente eficaz.

Consiste en aplicar el láser sin necesidad de contactar directamente sobre la superficie de una litiasis concreta sino mantener el láser pulsado de forma continua a alta frecuencia provocando un circuito con recorrido centrípeto de las litiasis que serán fragmentadas sin necesidad de mover la fibra (17).

Ésta técnica funciona mejor con litiasis de moderada o baja dureza y los autores recomiendan la combinación de $1 \mathrm{~J}$ y $20 \mathrm{~Hz}$ aunque nosotros solemos utilizar $1-1.4 \mathrm{~J}$ y $10 \mathrm{~Hz}$ para conseguir éste efecto.

Debemos tener en cuenta el principio demostrado por Sampaio y Aragao sobre el aclaramiento litiásico (18).

Es claro que a mayor tamaño de la litiasis tratada, el porcentaje de litiasis residual es mayor, pero consideramos que es un error evaluar el resultado en el periodo postoperatorio inmediato, tal y como se puede hacer en la NLP.

Tras el tratamiento de la litiasis con URS Flex hay que esperar unos meses tal y como se realiza al evaluar algunos casos de LEOC. La litiasis fragmentada no tiene porqué ser eliminada de inmediato al pulverizar gran parte de la misma o dejar mínimos fragmentos visibles en pruebas de imagen y hay que esperar al menos 1-2 meses para saber cual ha sido el verdadero éxito del tratamiento tras el aclaramiento litiásico espontáneo.

Así se explica la mejora en el porcentaje libre de litiasis en nuestra serie tras ser evaluado de inmediato y a los 3 meses de la cirugía $(77 \%$ y $92.7 \%)$. El catéter doble J puede ser de ayuda a la hora de facilitar la expulsión de esos fragmentos litiásicos residuales.

Respecto a la actitud frente a los pacientes que quedan con fragmentos residuales, aplicamos lo que se recoge en las Guidelines de Urolitiasis de la Asociación Europea de Urología 2010 (14. Fragmentos Residuales, tabla 25), el seguimiento es la opción de elección para los fragmentos residuales de menos de $5 \mathrm{~mm}$ en pacientes asintomáticos.
Como ya hemos comentado, nuestros pacientes permanecen la mayoría con el catéter doble J durante 3 semanas de forma postoperatoria.

Aunque reconocemos que 3 semanas pueda ser excesivo y quizás con una semana sería suficiente, la planificación de las revisiones en consulta en nuestro Servicio hacen difícil poder ser valorados antes y retirarles el catéter.

Una de las principales limitaciones de la URS Flex es una anatomía desfavorable como se describe en el artículo de Geavlete donde explican la influencia de la anatomía pielocalicial en el éxito de la URS Flex (19).

En nuestro caso, y gracias a la experiencia acumulada en la NLP desde 1986 en nuestro Servicio, creemos que una limitación importante serían los pacientes con antecedente de cirugía abierta previa (pielonefrolitotomía abierta), ya que muchos de éstos pacientes quedan con una cierta retracción piélica y fibrosis residual, que no permitiría una correcta distensión de la vía urinaria con la irrigación de suero y por tanto ausencia de espacio para trabajar con comodidad. Es lo que hemos observado al realizar NLP en pacientes con éste tipo de antecedente quirúrgico aunque no tenemos experiencia con la URS Flex en éstos casos.

Smith y Patel evaluaron el impacto de la URS Flex en el manejo de la nefrolitiasis verificando su beneficio en pacientes obesos o con diátesis hemorrágica como alternativa frente a la LEOC o NLP.

Resaltan el posible efecto negativo en determinados casos en los que el tratamiento se limita a vaporizar la litiasis en algunos pacientes donde el problema fundamental es anatómico y que al igual que la LEOC fracasan ya que se puede fragmentar la litiasis pero al no eliminarse se vuelven a consolidar tal y como refleja Holland y cols. $(20,21)$.

Hay que subrayar que aunque el rango libre de litiasis es menor que el de la NLP, el coste, la invasividad y el número e importancia clínica de sus complicaciones lo hace superior en un diámetro a tratar en torno a los $2 \mathrm{cms}$.

Nosotros pensamos por el momento que si la litiasis puede ser eficazmente tratada en un solo acto quirúrgico, el grado de aceptación y satisfacción del paciente puede ser mayor aunque la agresión quirúrgica sea mayor.

Por tanto, litiasis mayores a $2 \mathrm{~cm}$ las trataremos con NLP y solo realizaremos URS Flex si la litiasis 
es de baja densidad radiológica (facilidad y rapidez en la litofragmentación) y la anatomía del paciente es favorable para una correcta eliminación espontánea de fragmentos litiásicos residuales (Tabla II).

En Litiasis entre $1-2 \mathrm{~cm}$ realizamos URS Flexible como primera opción terapéutica.

Aunque creemos que éste es un buen algoritmo terapéutico, sabemos que ha de ajustarse a las condiciones especiales de cada centro como la disponibilidad de LEOC o rendimiento en su litofragmentación lo cual pudiera alterar la mejor indicación en cada caso.

Otro campo interesante es el de los pacientes en edad infantil. La URS Flex ha demostrado ser una alternativa a la LEOC o al menos una posibilidad terapéutica válida con alta eficacia y baja morbilidad como lo demuestra con unos resultados SFR del $88 \%$ Corcoran en 2007 con 47 pacientes y una edad media de 9,4 años o Kims y cols con 170 pacientes de edad media 62,4 meses y SFR $100 \%$ en menos de $1 \mathrm{~cm}$ y $97 \%$ en litiasis mayores a $1 \mathrm{~cm}$ con un $100 \%$ tras un segundo procedimiento $(22,23)$.

No solamente se ha demostrado seguro en pacientes pediátricos sino también en embarazadas (24) y en pacientes anticoagulados o antiagregados como lo demuestra la serie de Turna con 37 pacientes y solo 3 de ellos presentaron hematuria postoperatoria que no precisó trasfusión (25).

\section{TABLA II.}

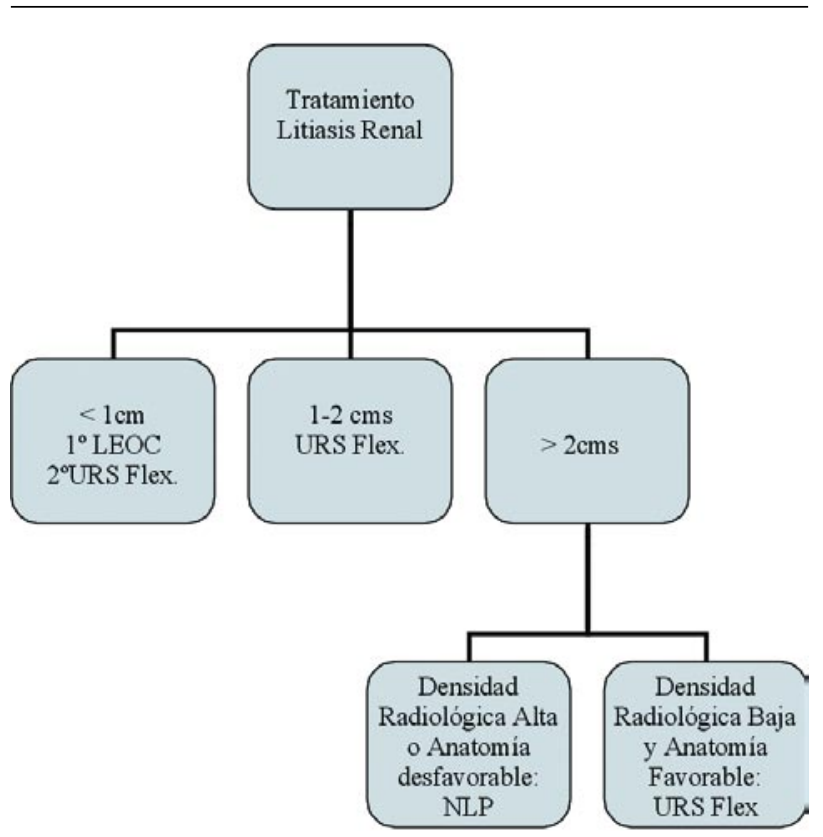

En determinados casos el presentar un catéter preoperatorio puede facilitar la cirugía, el paso de la vaina protectora y la extracción de fragmentos aunque esto no justifica la necesidad de cateterizar previamente a todos los pacientes como exponen los autores Shields y cols. (26).

Hyams y Shah que realizan una comparación de costes y resultados entre NLP y URS Flex con litiasis renales entre $2-3 \mathrm{~cm}$ comprobando que una URS Flex es menos costoso que la NLP e igualmente válido respecto a sus resultados (27).

\section{CONCLUSIÓN}

Como conclusión podemos defender el tratamiento de las litiasis renales mediante URS Flex hasta un tamaño máximo de $2 \mathrm{~cm}$ siguiendo nuestro algoritmo terapéutico.

Consideramos que la URS Flex en manos expertas puede ser una alternativa segura a la NLP en determinados casos de litiasis de mayor tamaño.

\section{BIBLIOGRAFIA y LECTURAS

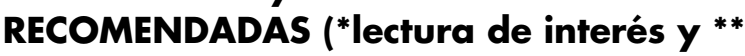 lectura fundamental)}

1. Marshall VF. Fiber optics in urology. J.Urol, 1964; 91:110-4.

2. Takayasu H, Aso Y, Takagi T, Go T. Clinical application of fiber-optic pyeloureteroscope. Urol Int, 1971; 26: 97-104.

3. Bagley DH, Huffman JL y Lyon ES. Flexible ureteropyeloscopy: diagnosis and treatment in the upper urinary tract. J Urol, 1987; 138: 280-285.

4. Bagley DH y Rittenberg MH: Intrarenal dimensions. Guidelines for flexible ureteropyeloscopes. Surg Endosc 1987; 1: 119-121.

5. Aso Y, Ohta N, Nakano M, Ohtawara Y, Tajima A y Kawabe K. Treatment of staghorn calculi by fiberoptic transurethral nephrolithotripsy. J Urol 1990;144: 17-19.

*6. Lésperance J, Ekeruo W, Scales C Jr, Marguet C, Springhart P, Maloney M y cols. Effect of ureteral access sheath on stones-free rates in patients undergoing ureteroscopic management of renal calculi. Urology 2005; 66: 252-255.

7. Pearle MS, Lingeman JE, Leveillee R, Kuo R, Preminger G, Nadler R y cols. Prospective, randomized trial comparing shock wave lithotripsy and ureteroscopy for coger pole caliceal calculi 1 cm or less. J Urol, 2008; 179(5 Suppl):S69-73. 
8. Wong M. Flexible Ureteroscopy is the ideal choice to manage a $1.5 \mathrm{~cm}$ diameter lower-pole stone. $\mathrm{J}$ Endourol, 2008; 22: 1845-6.

*9. Grasso M, Ficazzola M. Retrograde ureteropyeloscopy for lower pole caliceal calculi. J Urol, 1999; 162: 1904-8.

10. Chung B, Aron M, Hegarty N, y Desay M. Ureteroscopic versus percutaneous treatment for medium-size (1-2-cm) renal calculi. J Endourol, 2008; 22: 343-6.

*11. Breda A, Ogunyemi O, Leppert J, Lam J y Schulam P. Flexible ureteroscopy and laser lithotripsy for single intrarenal stones $2 \mathrm{~cm}$ or greater-is this the new frontier?. J Urol, 2007; 179: 981-4.

12. El-Anany FG, Hammouda HM, Maghraby HA y Elakkad MA. Retrograde ureteropyeloscopic holmium laser lithotripsy for large renal calculi. BJU Int, 2001; 88: 850-

13. Miller NL y Lingeman JE. Management of Kidney stones. BMJ 2007;334:468-

14. Mc Dougall E. Percutaneous approaches to the upper urinary tract. Campbell's Urology, $8^{\mathrm{a}}$ ed. Editada por PC Walsh, AB Retik, ED Vaughan Jr, AJ Wein, LR Kavoussi, AC Novick y cols. Philadelphia: WB Sanders Co 2002: 3320-3360.

**15. Breda A, Ogunyemi O, Leppert J y Shulam P. Flexible ureteroscopy and laser lithotripsy for multiple unilateral intrarenal stones. Eur Urol, 2009; 55: 1190-7.

**16. Mariani AJ. Combined electrohydraulic and holmium:YAG laser ureteroscopic nephrolithotripsy of large (greater than $4 \mathrm{~cm}$ ) renal calculi. J Urol, 2007; 177:168-73.

*17. Chawla S, Chang M, Chang A, Lenoir J y Bagley D. Effectiveness of High-frecuency holmium: YAG laser stone fragmentation: the "popcorn effect". J Endourol, 2008; 22: 645-9.

18. Sampaio FJ y Aragao AH. Limitations of extra- corporeal shockwave lithotripsy for coger caliceal stones: anatomic insight. J Endourol, 1994; 8: 241-

*19. Geavlete P, Multescu R, y Geavlete B. Influence of pyelocaliceal anatomy on the success of flexible ureteroscopic approach. J Endourol 2008; 22: 2235-2239.

20. Smith RD y Patel A. Impact of flexible ureteroscopy in current management of nephrolitiasis. Curr Opin Urol 2007; 17: 114-119.

21. Holland R, Margel D, Livne PM Lask DM, Lifshitz D. Retrograde intrarenal surgery as secondline therapy yields a lower success rate. J Endourol 2006;20: 556-559.

22. Smaldone M, Corcoran A, Docimo S y Ost M. Endourological management of pediatric stone disease: present status.J Urol 2009; 181: 17-28.

23. Kim S, Kolon T, Canter D, White M y Casale P. Pediatric flexible ureteroscopic lithotripsy: the children's hospital of Philadelphia experience.J Urol 2008; 180: 2616-2619.

24. Akpinar H, Tufek I, Alici B y Kural AR. Ureteroscopy and holmium laser lithotripsy in pregnancy: stents must be used postoperatively. J Endourol 2006; 175: 1080-1082.

25. Turna B, Stein R, Smaldone M, Santos B, Kefer J, Jackman S y cols. Safety and efficacy of flexible ureteroscopy and holmium:YAG lithotripsy for intrarenal stones in anticoagulated cases. J Urol 2008; 179: 1415-1419.

26. Shields J, Bird V, Graves R y Gómez-Marín O. Impact of preoperative ureteral stenting on outcome of ureteroscopic treatment for urinary lithiasis. J Urol 2009; 182: 2768-2774.

*27. Hyams E y Shah O. Percutaneous nephrolithotomy versus flexible ureteroscopy/Holmium laser lithotripsy: cost and outcome análisis. J Urol 2009; 182: 1012-1017. 